\title{
Musicoterapia para mujeres supervivientes de violencia de género y sus hijas e hijos Ana Gasco ${ }^{1}$
}

Recibido: 20/12/2020 Aceptado: 2/01/2021

RESUMEN: La violencia de género en la pareja y en la familia, constituye una de las principales causas de muerte para la mujer en todo el mundo. Las secuelas -tanto físicas, psicológicas como emocionales- de haber sufrido una relación de maltrato y abuso son realmente profundas y duraderas, no solo para la mujer que lo ha sufrido sino para las hijas e hijos que están expuestas a ello. Hasta hace bien poco, los menores no eran considerados víctimas de la violencia de género, aunque no cabe duda de que ya sea de forma directa o indirecta deja una importante huella emocional y psicológica en ellos. A través de la musicoterapia trabajaremos con ambos colectivos para la liberación emocional, la recuperación de la identidad y el refuerzo del vínculo materno filial, de fundamental importancia para el desarrollo del menor.

Palabras Clave: violencia de género, violencia contra la mujer en la pareja, musicoterapia, menores expuestos a violencia de género, intervención terapéutica.

\section{Music therapy for women survivors of gender violence and their daughters and sons}

ABSTRACT: Gender-based violence in couples and families is one of the leading causes of death for women
worldwide. The consequences - physical, psychological and emotional - of having suffered an abusive relationship
are truly profound and lasting, not only for the woman who has suffered it but also for the children who are
exposed to it. Until recently, children were not considered victims of gender violence, although there is no doubt
that either directly or indirectly it leaves a significant emotional and psychological mark on them. Through music
therapy we will work with both groups for the emotional liberation, the recovery of identity and the reinforcement
of the maternal-filial bond, of fundamental importance for the development of the minor. Key words: gender-based violence, domestic violence, music therapy, children exposed to domestic violence, therapeutic intervention.

Cómo citar: Gasco, A. (2020). Musicoterapia para mujeres supervivientes de violencia de género y sus hijas e hijos. Revista de Investigación en Musicoterapia. 4, 21-43. https://doi.org/10.15366/rim2020.4.002

\footnotetext{
${ }^{1}$ Musicoterapeuta y profesora en la Escuela de Música de Humanes (Madrid) anapercusion@gmail.com
} 


\section{INTRODUCCIÓN}

\section{Justificación y problema}

Vivimos en una sociedad patriarcal de considerable naturalización de los abusos hacia la mujer. Este contexto sociopolítico e ideológico da lugar a la fuerte desigualdad y asimetría que existe en las relaciones entre mujeres y hombres, facilitando así la violencia de género (Expósito, 2011). La relación de sumisión y dependencia de la mujer con el hombre durante décadas (Hirigoyen, 2006), los estereotipos de género (Fernández, 2004), el rol de la mujer en la sociedad y la idea del amor romántico (Samaniego, 2020) entre otras creencias y mitos, dificulta y complica la visualización y conceptualización de este problema que se oculta tras la desvalorización de la mujer. En muchos casos haciendo que esta violencia de género (VG) pase desapercibida, se oculte, sea indiferente o se considere normal (Expósito, 2011; Romero, 2015).

$\mathrm{El}$ aumento exponencial de la violencia contra la mujer $(\mathrm{VcM})$ es una realidad latente y de consecuencias devastadoras en nuestra sociedad. Constituye un problema de salud pública así como una violación de los derechos humanos de la mujer (OMS 2013). En el mundo, una de cada tres mujeres (35\%) ha sufrido VG alguna vez en su vida y unas 137 mujeres son asesinada cada día por sus parejas, según cifras de las Naciones Unidas en 2017. El problema de la VG es creciente en todos los contextos sociales y culturales, sin que haya distinción de edad, clase social, nivel de estudios, raza o religión (Walker, 1979; Whipple y Lindsey, 1999). Es un conflicto que afecta a la mujer y en consecuencia a sus descendientes ya sea de forma directa o indirecta, dejando en España a 46 niñas y niños huérfanos menores de 18 años en 2019 (Delegación del Gobierno para la violencia de género 2019).

Las mujeres que han sufrido maltrato se ven afectadas por severos problemas de salud, tanto a nivel físico como a nivel psicológico-emocional (Whipple y Lindsey, 1999; Barreiro 2018, Lago 2012), al igual que los menores que han vivido y sufrido estos contextos de VG. La necesidad de rehabilitación, no solo individual de ambos, sino también, del vínculo materno filial (Rey, 2016) es lo que trataremos en esta propuesta de intervención. El miedo, el aislamiento social, y la pérdida de identidad que sufren estas mujeres, les lleva a una fuerte desconexión con ellas mismas, con sus hijas e hijos y con la vida exterior. Por otro lado, las hijas e hijos que han sido testigos de VG en relación a su madre, tienen un alto riesgo de alteración en su desarrollo integral, menos rendimiento escolar, problemas en sus habilidades sociales y un porcentaje considerable de perpetuar esta violencia en el futuro (Fernández et al., 2003).

Desde la Antigüedad, la música ha sido un instrumento para paliar, curar y evitar los males (Jauset 2013), llegando a lo más profundo de nuestro ser. La musicoterapia, nos ayuda a trabajar nuestras emociones, a conectar con nosotros mismos, a comunicarnos y a expresarnos con mayor facilidad. Una forma amable y cuidadosa de ahondar en esas emociones enquistadas y tan dolorosas de estas mujeres y sus hijas e hijos, que han vivido una situación tan malvada e injusta. Esta propuesta de intervención pretende crear un espacio de escucha, de respeto, de comprensión, de valorización, de afecto, a través de la musicoterapia. Un espacio tanto para las madres como para las hijas e hijos donde poder RE-conocerse, RE- conciliarse y REcrearse. 


\section{Objetivos}

Objetivos generales:

- Recuperar la identidad propia y el empoderamiento a través de la musicoterapia.

- Sanar y reforzar el vínculo materno filial.

- Evitar el desarrollo transgeneracional de conductas violentas y abusivas.

Objetivos específicos mujeres:

- Disminuir los niveles de ansiedad y estrés para una mayor relajación física y psicológica a través de la música.

- Reconocer y liberar las emociones y sentimientos ocultados y bloqueados mediante la escucha y la participación musical.

- Trabajar la autoestima y el autoconocimiento por medio de la improvisación musical y la composición.

Objetivos específicos hijas e hijos:

- Reconocer y liberar las emociones y sentimientos bloqueados mediante la escucha y la participación musical.

- Fomentar la interrelación positiva, empática, segura y de respeto que se crea en los trabajos musicales en grupo.

- Promover el aprendizaje desde una perspectiva feminista e igualitaria.

\section{MARCO TEÓRICO}

\section{Violencia de Género o Violencia contra la mujer}

Normalmente los términos de violencia de género, violencia contra la mujer, violencia machista o violencia doméstica entre otros, se usan para nombrar el abuso y maltrato del hombre hacia la mujer en un contexto íntimo, familiar y de pareja. Sin embargo, este concepto es mucho más amplio y engloba todo tipo de violencia ejercida hacia la mujer por el mero hecho de ser mujer. En el artículo 2 de la Declaración sobre la eliminación de la violencia contra la mujer de Naciones Unidas (1993) se incluye no sólo la violencia en la relación de pareja, si no también cualquier abuso o maltrato a mujeres y niñas en el entorno familiar, el acoso y el abuso sexual en el trabajo, la trata de mujeres, la prostitución forzada, la mutilación genital femenina o cualquier tipo de violencia perpetrada o tolerada por el Estado entre otras. Aunque en España las expresiones generalmente aceptadas y mas utilizadas puedan ser violencia de género o violencia machista para denominar este problema, las discusiones y los cambios sobre la terminología han sido uno de los puntos más debatidos en los discursos sobre violencia contra la mujer desde que empezó a tratarse (Fernández, 2015). Dependiendo del análisis de cada autor, el punto de mira se pondrá en diferentes aspectos para definir y nombrar la violencia ejercida hacia la mujer. Lo que queda claro es que no podemos olvidarnos de las connotaciones sociales, políticas y culturales en las que esta violencia se genera y se resguarda. Por todo ello, en esta propuesta de intervención utilizaremos los término de violencia de género en la pareja, VGP, o violencia contra la mujer en la pareja,VcMP, considerándolo como la 
violencia ejercida por el hombre sobre la mujer en una situación de pareja o expareja, al igual que violencia de género en el núcleo familiar, VGF, teniendo en cuenta la exposición y la transmisión de esta violencia en las y los menores descendientes. Los términos han sido elegidos cuidadosamente de forma que, violencia de género es frecuentemente la palabra usada en España y como su propio nombre indica en la Ley Integral contra la Violencia de Género aprobada en 2004; y el término violencia contra la mujer es el utilizado por la ONU en su Declaración sobre la Eliminación de la Violencia contra la Mujer de 1993, y que se cita a continuación. Según Naciones Unidas, la violencia contra la mujer se define como " todo acto de violencia de género que resulte, o pueda tener como resultado un daño físico, sexual o psicológico para la mujer, inclusive las amenazas de tales actos, la coacción o la privación arbitraria de libertad, tanto si se producen en la vida pública como en la privada".

Como podemos observar tras esta definición, cuando hablamos de maltrato hacia la mujer, suele clasificarse en tres grupos (Labrador, Rincón, De Luis y Fernández-Velasco, 2004; Lago, 2012; Fernandez et al, 2003):

1. Violencia física: Cualquier conducta o procedimiento de forma intencional que cause o pueda causar daño físico en la otra persona. Es el tipo más evidente de violencia y el más fácil de identificar.

2. Violencia psicológica: Cualquier acto o conducta que tiene como intención intimidar, desvalorizar, culpar, humillar, etc.... a la mujer, ya sea en privado o en público, atentando contra su integridad psíquica y emocional. Esto incluye control económico, aislamiento social, insultos y amenazas entre otras. Es la violencia más difícil de identificar, ya que no produce un impacto social.

3. Violencia sexual: Cualquier acto sexual no consentido y forzado, incluyendo las agresiones, el acoso, el abuso, la violación y la explotación sexual.

Pero hay algunas otras clasificaciones que añaden un último punto y que resulta interesante remarcar, es la Violencia del Sistema, Systemic Violence (Curtis, 1996) que es la que resulta del fracaso y la ineficacia de los servicios legales, sociales y de salud.

\section{Violencia de género en la pareja y en el núcleo familiar}

Podríamos definir la violencia de género en el núcleo familiar, como todo tipo de abuso, ya sea físico, psicológico y/o verbal, basado en la dinámica de poderes para controlar las relaciones emergentes (Fernández, 2004). Como manifiestan Amor, Echeburúa, de Corral, Zubizarreta y Sarasua (2002):

Por extraño que pueda parecer, el hogar -lugar, en principio, de cariño, de compañía mutua y de satisfacción de las necesidades básicas para el ser humano - puede ser un sitio de riesgo para las conductas violentas (...) Lo que lleva a la violencia es la percepción por parte del agresor de la vulnerabilidad y de la indefensión en que se encuentran las víctimas en el hogar (mujeres, niños y ancianos) y la sensación de impunidad (...) En los hogares donde hay mujeres maltratadas suele haber también niños maltratados (p. 229).

Esta violencia conlleva importantes riesgos para la salud de las mujeres tanto a nivel físico como psicológico, generando un fuerte impacto emocional que crea una situación de desequilibrio para la salud mental de las mujeres y de los posibles descendientes o familiares que vivan bajo estas circunstancias (Campbell et al., 2002). Los estereotipos sociales acerca del papel de la mujer y de las relaciones de pareja desempeñan un papel determinante en el 
mantenimiento de este tipo de violencia (Lorente, 2001). Como planteaba Dohmen (1992, p. 65 , cit. por Fernández, 2004, p. 164):

La violencia es una forma de control que se apodera de la libertad y la dignidad de quien la padece. El maltrato intrafamiliar es una de las manifestaciones que refleja la dominación masculina y la subordinación femenina. El abuso es una conducta que efectiviza un grave descenso en la autoestima, impidiendo el desarrollo de la persona como un sujeto independiente.

La VGF a diferencia de otras violencias contra la mujer, tiene unas características concretas (Amor et al., 2002):

- Es una conducta que no suele denunciarse, aunque cada vez más.

- Es una conducta continuada en el tiempo, mayoritariamente el maltrato se establece durante el noviazgo o en el primer año de matrimonio.

- En un gran porcentaje el maltrato se extiende a las hijas e hijos, corriendo el riesgo de ser una conducta aprendida, lo que conlleva una transmisión cultural de los patrones aprendidos

"Las investigaciones llevadas a cabo en los últimos 25 años han puesto de manifiesto la existencia de una estrecha asociación entre la violencia en la pareja y el maltrato infantil" (Patró y Limiñana, 2005, p.12).

Todas nos preguntamos cómo puede ser que una mujer que está siendo sometida a tanta violencia y humillación, no sea capaz de denunciar y abandonar a su agresor. La depresión, la ansiedad extrema, la pérdida de autoestima y los sentimientos de culpabilidad entre otras causas, dificultan notablemente la salida de estas relaciones de control y agresión. Algunos factores comunes que se han identificado para explicar por qué una mujer no denuncia o pide ayuda son (Zubizarreta, 2004):

- Alta tolerancia a los comportamientos violentos y temor a las consecuencias relacionadas con la separación.

- Dependencia de la mujer respecto a su pareja, afectiva y social, aunque a veces también económica. La VGP conlleva un aislamiento social importante, favoreciendo al agresor como única fuente cercana, aumentando así el control sobre la mujer.

- Miedo a represalias por parte del maltratador.

- Estado psicológico de la mujer.

Además de esto, hay que entender que la violencia suele presentarse de manera cíclica intercalándose con momentos de calma y cariño. La Dra. Leonore Walker describió en 1979 lo que se conoce como la teoría del círculo de la violencia, donde explica el proceso que la mujer vive y en el que llega a sentirse atrapada. El círculo tiene tres etapas:

> Primera fase de tensión. Donde los conflictos y las situaciones violentas van aumentando. La mujer trata de evitar comportamientos y/o acciones que puedan irritar a su agresor.

$>$ Segunda fase de agresión. La violencia se hace presente y se producen agresiones tanto físicas, psicológicas como sexuales. La mujer entra en un estado de miedo y ansiedad 
dando lugar en algunos casos a que se aísle y en otros a que se informe sobre posibles ayudas.

$>$ Tercera fase de conciliación o luna de miel. En esta fase se hace presente el arrepentimiento, las disculpas y las promesas por parte del agresor. Este cambio de actitud da esperanza a la mujer, creyendo en un posible cambio y solución del problema llegando así a la reconciliación.

Esta secuencia de acontecimientos supone una escalada constante de la violencia, ya que a medida que pasa el tiempo, la tensión y la tirantez crecen, aumentando así la probabilidad de un episodio violento. Por ello las mujeres que sufren maltrato, van elaborando diferentes estrategias de enfrentamiento y supervivencia durante la relación (Zubizarreta, 2004). Este desgaste físico y psicológico hace que lleguen a tales niveles de impotencia, anulación y desempoderamiento que pierden su propia identidad. "Terminas desconectando de ti misma, también fisicamente, porque no tienes energías ni fuerza para nada" (A better man, 2017). Una mujer que ha sufrido violencia se siente amenazada y denigrada afectando directamente a su capacidad para relacionarse, incluso con sus hijos (Formación Continua en Radio 3, 2019). Los vínculos interpersonales que crea una mujer con su bebé o anteriormente en el periodo de gestación, se pueden ver dañados notablemente. La visión idealiza de la maternidad junto con la frustración y desesperación por la violencia sufrida, puede dar lugar a sentimientos contradictorios que se reflejen en la crianza y en el bebé negativamente (Romero, 2015).

Como decíamos anteriormente, el riesgo de transmisión de conductas violentas y agresivas entre generaciones es alta, aunque no se puede hablar de una relación de causa efecto. Desde el año 2015, los menores son reconocidos en nuestro ordenamiento jurídico como víctimas directas de la violencia de género.(Art.1 apartado 2 Ley Orgánica 8/2015, de 22 de julio). Save the Children estima que 800.000 niños y niñas en España sufren las consecuencias de la violencia de género (2011).

"Los menores son víctimas de violencia cuando esta se ejerce sobre sus madres, porque la violencia no se encapsula, sino que irradia efectos a las personas que rodean, de forma más cercana, a la víctima inmediata, que es la mujer" (Gómez, 2018, p. 5).

Como avala Pinheiro en el Informe Mundial Sobre la Violencia Contra los Niños y las Niñas (2010):

Los niños y niñas en cuyo hogar se viven situaciones de violencia de género, son víctimas de violencia, ya que sufren de manera directa las consecuencias, no sólo físicas y emocionales que se detallan a continuación, sino también las derivadas de haber vivido y formado su personalidad en un ámbito de desigualdad de poder y sometimiento de la madre a la conducta violenta de un hombre, lo que potencialmente les convierte en elementos de la cadena de reproducción de esta violencia.

Al igual que el propio Tribunal Supremo declara en una de sus sentencias (Sentencia $n$. 247/2018, 24 mayo 2018):

(...) La presencia de los hijos e hijas en episodios de violencia del padre hacia la madre es una experiencia traumática, produciéndose la destrucción de las bases de su seguridad. Los menores [quedan] a merced de sentimientos de inseguridad, de miedo o permanente preocupación, ante la posibilidad de que su experiencia traumática vuelva a repetirse (...) desde luego, afecta muy negativamente al desarrollo de la personalidad del 
menor, pues aprende e interioriza los estereotipos de género, las desigualdades entre los hombres y mujeres, así como la legitimidad del uso de la violencia como medio de resolver conflictos familiares o interpersonales fuera del ámbito de la fami- lia.

Como podemos leer en la Convención sobre los Derechos del Niño (Unicef, 2006), la familia, definida de manera amplia, "es el medio natural para el crecimiento y bienestar de todos sus miembros" (pag. 8). La violencia a la que se ven sometidos las y los menores tanto directa como indirectamente, condiciona e interfiere en su desarrollo. Las agresiones, las humillaciones y la degradación a la que se ven sometidos o ven sometida a su madre, destruye los vínculos afectivos que son una parte fundamental de la niñez, y que están íntimamente unidos a la capacidad de amor y empatía y con el desarrollo de relaciones futuras. En muchas ocasiones las y los menores pueden ser utilizados por parte del padre agresor para seguir ejerciendo control y violencia sobre la madre, lo que obstaculiza totalmente su evolución, tanto en su cuidado como en la creación del vínculo materno filial. (Formación Continua en Radio 3, 2020). Las interacciones sucedidas en un contexto de miedo y desconfianza, como resultado de violencia parental y abuso, crean fuertes barreras personales y sociales. La historia de las interacciones ocurridas en el pasado va a condicionar el contexto y la forma en la que las personas se relacionan juntas en el presente (Pasiali, 2013). Por ello, debido a las experiencias vividas, muchos menores reaccionan con comportamientos de aislamiento y bloqueo, como medida de protección.

Holden (2003) separa en diez categorías los diferentes tipos de exposición de las y los menores a la violencia de género:

- Perinatal: el estrés sufrido durante el embarazo afecta al desarrollo del feto.

- Intervención: los menores se involucran para intentar parar el suceso violento, ya sea verbal o fisicamente.

- Victimización: los menores pueden ser dañados de manera intencional o accidental en los episodios de violencia.

- Participación: los menores pueden unirse obligados o de forma voluntaria a los episodios de violencia

- Testigo presencial: ver el episodio violento directamente.

- Escuchar: los menores no escuchan lo que ocurre directamente, pero escuchan las agresiones.

- Observación de los efectos de la agresión: los menores ven las consecuencias inmediatas de la agresión (moratones, heridas, objetos rotos...).

- Experimentar las consecuencias posteriores de la agresión: los menores experimentan cambios en su vida a consecuencia de las agresiones (cambio de residencia, convivir con la depresión de la madre, separación del padre...).

- Escuchar acerca del incidente: tomar conciencia de la agresión a través de otras personas.

- Desconocer los sucesos violentos: los menores no tienen conciencia de la agresión (los menores no están en el hogar, o la agresion ocurre fuera de casa...)

Como venimos exponiendo, el hogar es el lugar donde por primera vez, las niñas y niños experimentan las desigualdades basadas en el género y donde se reafirman o desafían las futuras relaciones de poder desequilibradas (Pinheiro, 2010; Fernández, 2004). Todas las formas de violencia en el hogar y la familia tendrán implicaciones profundas para el desarro- 
1lo, comportamiento y bienestar en la etapa adulta y para el desempeño como posibles progenitores en el futuro.

"Los niños aprenden a definirse a sí mismos, a entender el mundo y cómo relacionarse con él a partir de lo que observan en su entorno más próximo” (Patró y Limiñana, 2005).

\section{Sintomatología e Impacto}

La violencia que sufren las mujeres por parte de sus parejas o exparejas, al igual que la violencia que sufren sus hijas e hijos, ya sea directa o indirectamente, da lugar a importantes problemas de salud en ambos grupos (Ruiz-Pérez, Blanco-Prieto y Vives-Cases, 2004). A pesar de los modelos clasificatorios que tenemos de los síntomas o patologías, hay autoras que defienden que no se puede categorizar e ignorar el contexto propio e individual de cada mujer (Curtis, 2012).

\section{A. Mujeres que han sufrido VGP}

El cuadro clínico de cada mujer puede variar de unos casos a otros, sin embargo, podemos hablar de síntomas psicopatológicos y alteraciones en el comportamiento, que las mujeres que han sobrevivido a VGP normalmente presentan. Algunos estudios demuestran el incremento de ciertos síntomas hasta en un 50\% - 70\% (Campbell et al., 2002).

Se partirá de la hipótesis de que "las mujeres que han abandonado una relación de maltrato están en situación de riesgo debido a las secuelas que, a nivel cognitivo, emocional- afectivo y físico han provocado los efectos secundarios de la victimización de sus experiencias de maltrato" (Galego, 2015, p.134).

Normalmente nos remitiremos al trastorno de estrés postraumático y al malestar emocional (Amor et al, 2002). El Trastorno de Estrés Postraumático (TEPT) "es el desarrollo de síntomas específicos tras la exposición a uno o más eventos traumáticos" (DSM-V, APA 2014, p. 274). Algunos de estos síntomas son las reacciones disociativas (la mujer siente como si se repitiera el suceso, despersonalización), malestar psicológico intenso, reacciones fisiológicas intensas, evitación de pensamientos o recordatorios externos, alteraciones cognitivas negativas y del estado de ánimo (disminución del interés, incapacidad persistente de experimentar sensaciones positivas), alteración importante de la alerta (hipervigilancia, irritabilidad, comportamientos imprudentes, problemas de concentración) (DSM-V, APA 2014). Incluso, podemos hablar de un síndrome postraumático específico, como subcategoría del TEPT, que es el Síndrome de la Mujer Maltratada, Este término que utiliza por primera vez la Dra. Walker (1979), divide en ocho grupos los signos y síntomas psicológicos que padecen las mujeres que han sufrido violencia de género por parte de su pareja o expareja. Éstos son (Walker, 2012; Walker et al, 2017):

- Recuerdos recurrentes e intrusivos del suceso traumático.

- Hiper excitación y altos niveles de ansiedad.

- Dificultades cognitivas y cambios del estado de ánimo.

- Conducta elusiva y entumecimiento emocional.

- Relaciones interpersonales conflictivas.

- Distorsión de la imagen corporal y dolencias físicas y/o somáticas.

- Problemas sexuales. 
- Disociación.

El importante malestar psicológico intenso y prolongado unido a la violencia física, conlleva un deterioro considerable en la calidad de vida de estas mujeres, dando lugar a alteraciones clínicas muy significativas como (Zubizarreta, 2004; Amor et al, 2002; Hirigoyen, 2006; Fernández, 2004; Romero, 2015):

- Dolores y daños físicos (traumatismos, heridas, dolores musculoesqueléticos...).

- Trastornos inmunológicos.

- Trastornos neurológicos (tartamudeo, pérdida de audición, problemas de vista...).

- Problemas respiratorios, gastrointestinales y cardiovasculares.

- Trastornos psicosomáticos, dolores crónicos.

- Ansiedad, trastorno del sueño.

- Depresión, baja autoestima, autoinculpación, falta de autonomía.

- Aislamiento social, inadaptación.

- Trastornos sexuales y reproductivos (problemas ginecológicos, embarazos no deseados, trastornos menstruales, pérdida apetito sexual).

- Trastornos de la alimentación.

- Abuso de sustancias.

- Ideas suicidas.

Estos cuadros clínicos necesitan una evaluación cuidadosa y delicada. Son procesos que requieren mucho tiempo para su recuperación, ya que la baja autoestima y la desvalorización hace que pierdan el control personal, su libertad de decisión y su capacidad de acción. Las mujeres que han sufrido VGP necesitan un largo periodo para tomar conciencia, reaccionar y empezar a organizar las experiencias traumáticas que han sufrido (Formación Continua en Radio 3, 2019). "Todo ello lleva a una interferencia significativa en el funcionamiento social y laboral, a una pérdida de interés (...) desde el punto de vista lúdico e interpersonal y a un cierto embotamiento afectivo (...) para captar y expresar sentimientos de intimidad y ternura" (Belloch, Sandín y Ramos, 2009, p. 139).

\section{B. Menores expuestos a VGF}

Como numerosos estudios demuestran, la VGF tiene un fuerte impacto negativo en las niñas y niños que lo viven, incluso como testigos. Esto se manifiesta en diferentes problemáticas tanto a nivel físico, emocional, cognitivo y/o conductual (Suriá, Rosser y Villegas, 2014; Pinheiro, 2010). Incluso en el embarazo, el estrés al que está expuesta la madre debido a la violencia, puede afectar en el crecimiento del feto, partos prematuros, lesiones fetales e incluso abortos espontáneos (Save the children, 2011; Ruiz-Pérez, Blanco-Prieto y Vives-Cases, 2004). La exposición temprana a la violencia puede perjudicar al cerebro en su proceso de maduración. Si la exposición es prolongada, ya sea directa o indirecta, la alteración del sistema nervioso e inmunológico puede provocar limitaciones cognitivas, emocionales y sociales, facilitando así la aparición de enfermedades, lesiones y problemas sociales (Perry, 2001). La violencia contra las niñas y niños, así como vivir en un hogar donde se está expuesto a la violencia frecuente contra seres a los que quieres, puede ser un significativo factor para el desarrollo de enfermedades y de muerte durante la etapa adulta. A parte del abuso de sustancias como el alcohol u otras drogas, se relaciona también con enfermedades pulmonares crónicas, cáncer, depresión, enfermedades hepáticas, obesidad y problemas crónicos de salud 
reproductiva (Felliti et al, 1998). Por otro lado, es fundamental para el desarrollo de la personalidad del menor, poder desarrollar un sentimiento de seguridad y protección en las personas que te rodean. Esto nos remite a la corriente teórica del apego, que señala como fundamentales los patrones aprendidos en las primeras etapas, estableciendo a su vez, los vínculos intersubjetivos que refieren a la necesidad de conexión emocional y comprensión, que repercutirán en la estructura básica de la persona (Romero, 2015; Galán, 2015). Normalmente, cuando las y los menores toman conciencia de las circunstancias vividas y del miedo que han generado a una figura que se supone que tiene que protegerlos, se produce el derrumbamiento de todas las bases de su seguridad (Patró y Limiñana, 2005; Formación Continua en Radio 3, 2020).

Algunos de los síntomas más claros que podemos observar en los menores son los siguientes (Pinheiro, 2010; Romero, 2015):

- $\quad$ TEPT

- Disminución de la capacidad cognoscitiva.

- Depresión, ansiedad, miedo.

- Retraso del desarrollo.

- Alteración en la regulación afectiva y los impulsos.

- Alteraciones en los sistemas de valores.

- Alteraciones en la concentración y la atención.

- Trastornos de la alimentación y el sueño.

- Comportamientos delictivos, violentos.

- Somatizaciones.

- Abuso de alcohol y otras drogas.

- Lesiones físicas.

Las niñas y niños pequeños, considerando esto antes de la edad de 6 años, son propensos a reexperimentar el trauma y los síntomas a través del juego. Por ello pueden darse conductas evasivas y/o restrictivas en torno al juego y a las actividades exploratorias. (DSM-V, APA, 2014). Por otro lado, en etapas más juveniles y adolescentes, la búsqueda de emociones fuertes, las conductas de alto riesgo o las conductas agresivas, pueden interferir en el comportamiento escolar y social con sus iguales (DSM-V, APA 2014), obstaculizando las relaciones con los demás y dando lugar al aislamiento, o a la unión de grupos potencialmente conflictivos (Patró y Limiñana, 2005).

Otro punto importante a tener en cuenta en las y los menores expuestos a la violencia de genero son los duelos. Las nuevas situaciones a las que se enfrentan o los cambios de rutinas habituales a los que suelen verse sometidos, requieren un tiempo para ser procesados y así asumir y asimilar que su vida ha cambiado. Hablamos por ejemplo de los cambios de residencia o de colegio, la pérdida de amigos y compañeros, la separación o divorcio de los padres... (Romero, 2015).

\section{Aplicación de la Musicoterapia en mujeres maltratadas}

Tomaremos como referencia algunos de los estudios más importantes que se han hecho con este colectivo hasta el momento. 
- Cassity and Theobold (1990). Este estudio se hace con un total de 80 musicoterapeutas, de los cuales 45 trabajaron con mujeres maltratadas, 61 con las hijas e hijos de mujeres maltratadas, y 28 con hombres maltratadores. Para los dos colectivos que tratamos en esta propuesta se utilizaron diversas técnicas como el análisis de letras de canciones y su discusión, la improvisación instrumental, el canto, la composición de canciones, el dibujo a través de la música y la expresión corporal. Todo esto ayudó al reconocimiento y expresión de los sentimientos, mayor relajación muscular, disminución de la ansiedad, y un aumento de la sociabilizancion.

- Whipple and Lindsey (1999). Es una investigación de referencia en la que se aborda una propuesta de musicoterapia para mujeres maltratadas. En ella recalcan la necesidad de empoderamiento, de mejora de la autoestima, de confianza y mayor asertividad de estas mujeres. El trabajo se hace en sesiones grupales debido a la importante correlación entre el apoyo social y el bienestar psicológico. A través del canto, la escucha de música, el análisis de letras, la improvisación y el uso de instrumentos, van desarrollando las sesiones y acercándose a sus objetivos, el aumento del nivel de relajación, la interacción social y la expresión individual, la identificación de sentimientos y planificación de futuras metas, eran algunos de ellos.

- Hernández-Ruiz (2005). Importante estudio en el que se demuestra que la musicoterapia constituye un método efectivo en la reducción del nivel de estrés y en la mejora de la calidad del sueño en mujeres maltratadas que están viviendo en los albergues (shelters) destinados para ellas. La música, elegida por las participantes, se escuchaba conjuntamente con una técnica de relajación muscular progresiva.

- Fernández (2004, 2006, 2010, 2013, 2015a, 2015b). Desarrolla varios trabajos en Cuba, Mexico y Baja California centrados en la autoestima, la ansiedad y la reconstrucción de la identidad en mujeres que han sufrido maltrato. En todos observamos una mejora de los aspectos estudiados y la relación tan importante que hay entre estos parámetros. A través de la audición musical, el trabajo corporal, el trabajo con canciones, la improvisación colectiva, el canto y la técnica de relajación de Schultz, se obtienen resultados muy positivos. Cabe destacar el uso de la ficha musicoterapéutica que nos permite conocer la historia de vida sonoro-musical de cada participante y que relacionamos con el concepto de ISO de Benenzon; segundo, la repetición de los patrones sonoros-musicales de la familia de origen; y tercero el trabajo que realiza con la canción infantil o canción de cuna, como evocación del arrullo infantil para volver a sentir el contacto como algo placentero y reconfortante.

- Curtis $(2012,2015)$. Aborda la práctica de la musicoterapia con mujeres que han sobrevivido a abusos, desde una perspectiva feminista, social y multicultural. Persevera en la importancia de tener en cuenta todos los aspectos que rodean a la mujer, esto incluye: edad, discapacidad, religión, etnia, clase social, orientación sexual, origen y género/sexo entre otras. Los métodos y procedimientos que utiliza son diversos. Dentro de la musicoterapia receptiva incluye la relajación muscular progresiva, GIM, y el análisis de letras de canciones; y en musicoterapia activa, actividades de improvisación, composición de canciones y su grabación, cantar, el uso de tambores y las audiciones. 


\section{Aplicación de la Musicoterapia en menores expuestos a VGF}

Hay pocos estudios del uso de la musicoterapia en menores expuestos a violencia de género en la familia, así que tomaremos como referencia los expuestos:

- Pasiali (2013). Trabaja en la recuperación del vínculo materno filial, de dos niñas menores y su madre. En este caso tanto la madre como la mayor de las hermanas, habían sufrido una relación de maltrato y abuso por parte del marido y padre. Reducir el estrés de la niña, aprender a regularse, recuperar la confianza en las relaciones con adultos y la comunicación entre ellas, son alguno de los objetivos que se presentan en este estudio.

- Annesley (2015). Nos muestra el estudio que se lleva a cabo con niñas, niños y adolescentes expuestos a violencia de género familiar. Realiza un estudio cualitativo de los efectos de las intervenciones musicoterapeúticas, dando resultados positivos. Se realizan entrevistas tanto a los menores como a las madres y padres y otros cuatro profesionales no musicoterapeutas, adjuntos al proyecto.

\section{PROPUESTA DE INTERVENCIÓN}

Esta propuesta de intervención tendrá una duración total de 5 meses que dividiremos en dos bloques, estructurados de la siguiente forma:

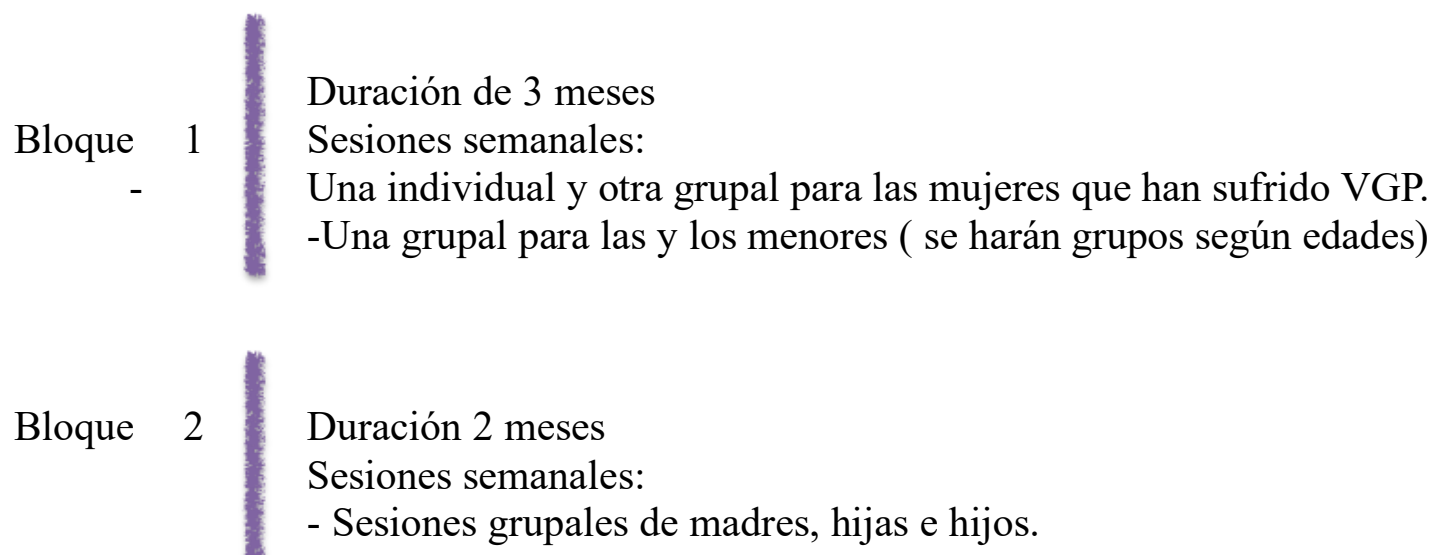


- Necesitaremos un espacio amplio y tranquilo, donde podamos desarrollar las sesiones sin interrupciones, sintiéndose seguras y $\sin$ distracciones ni ruidos externos.

- Contaremos con una silla y una esterilla para el suelo por cada participante y Recursos musicoterapeuta.

- Instrumentos variados de percusión melódicos y rítmicos, como xilófonos, metalófonos, campanas afinadas, crótalos, pandero y pandereta, cascabeles, maracas y shaker, triángulo, ocean drum, palo de lluvia, plato suspendido, templeblocks o caja china, dejmbe o darbuka, guitarra y piano... cuanta más variedad instrumental, mayor riqueza tímbrico-sonora.

- Un reproductor de música con sistema de altavoces.

- Una grabadora de audio.

Diversos autores hablan de mayores beneficios de un tratamiento doble de sesiones individuales y de sesiones grupales, aunque siempre remarcan la importancia de las sesiones grupales para romper el aislamiento al que se han visto sometidas las mujeres por su agresor (Echeburúa y de Corral, 1998; Curtis, 1996; Whipple y Lindsey, 1999).

A lo largo de las sesiones utilizaremos diferentes técnicas y actividades que han sido ya investigadas con resultados muy positivos como los que comentábamos en los estudios propuestos anteriormente. Cantar, tocar instrumentos, audiciones musicales, composición de canciones, improvisación, danza y movimiento corporal, relajaciones guiadas, etc.

En este trabajo se presentará detalladamente sólo la propuesta grupal para las mujeres que han sobrevivido a la VGP, siendo la siguiente:

$>$ Duración

El programa se estructurará a lo largo de 3 meses, con un total de 12 sesiones semanales grupales de 90 minutos. A esto se le añadirá una sesión de evaluación inicial, donde conocernos entre todas, explicar la propuesta y escuchar sus ideas o peticiones, y trabajar la ficha musicoterapeutica o de historia musical.

$>$ Recogida de datos

Haremos un estudio cualitativo a través de entrevistas personales que se harán en las sesiones individuales. Estas sesiones se dividirán en el trabajo individual musicoterapeutico y la entrevista. Igualmente, a lo largo de las sesiones grupales se irán pasando diferentes pruebas de evaluación, como el Inventario de Autoestima de Coopersmith (1967), la Escala Sintomática de Estrés de Seppo-Aro (1980), el Inventario de Ansiedad Estado-Rasgo (STAI) (Spielberger, Gorsuch y Lushene, 1970) para un análisis cuantitativo de la sesión.

$>$ Sesiones de Musicoterapia

El contenido de cada sesión variará según las necesidades que vayan surgiendo en el grupo. Haremos un encuadre detallado de los métodos y actividades a realizar, que se podrán Revista de Investigación en Musicoterapia, 4, 2020, pp. 21-43 https://doi.org/10.15366/rim2020.4.002 
combinar y presentar conforme el grupo vaya evolucionando. "La flexibilidad resulta precisa en cualquier protocolo de intervención, pero más aún en el caso de las víctimas de maltrato"(Echeburúa y de Corral, 1998, p.52). Utilizaremos musicoterapia receptiva como las actividades de relajación, audición y análisis lírico; y musicoterapia activa como tocar instrumentos y cantar.

\section{Actividades de relajación y visualización}

Empezaremos siempre con trabajos de relajación corporal, de respiración y de visualización guiada. A largo plazo cumplirá uno de nuestros objetivos que es disminuir la ansiedad y el estrés, pero a la vez nos ayudará en cada sesión a centrase, a conectar consigo mismas, a dejar de lado el ajetreo del día y a conseguir una atención plena en la sesión. Estos ejercicios son realmente importantes para su posterior aplicación fuera de las sesiones. Alentaremos a las participantes a tumbarse en el suelo y cerrar los ojos, siempre que les resulte cómodo y se sientan seguras, para una percepción corporal mas profunda. Utilizaremos la técnica de relajación muscular progresiva de Jacobson, que se centra en la tensión y distensión de los músculos; la técnica de relajación de Schultz, que traslada al cuerpo a diferentes sensaciones y focalizaciones. Trabaja con la sensación de pesadez en el cuerpo, sensación de calor, la concentración en los latidos del corazón tan importante para estas mujeres que necesitan escuchar su fuerza interior y conectar consigo mismas, la respiración para ir desahogando y soltando la angustia, y por último el abdomen considerado como el segundo cerebro en las culturas orientales. Las visualizaciones guiadas se irán introduciendo poco a poco, ya que pueden producir cierto rechazo al principio. Las utilizaremos para aumentar el nivel de relajación, de conciencia y de desconexión con el exterior. Serán paseos guiados por escenarios tranquilos, naturales, de amplitud, donde se sientan protegidas y puedan refugiarse en cualquier momento que lo necesiten. En ellas también trabajaremos la expresión de los sentimientos que van surgiendo en los paseos a través de los instrumentos.

Todos estos ejercicios irán acompañados normalmente de música grabada, mucha de ella elegida por las participantes para que el efecto de relajación tenga una mayor duración (Hernández-Ruiz, 2005). Ésta se entregará recopilada en un CD para el trabajo personal fuera de las sesiones. En las sesiones la musicoterapeuta también podrá utilizar música en vivo si así lo considera. Una vez finalizado esta primera parte, se hará una exposición-coloquio de las sensaciones vividas, de las facilidades o dificultades que se han encontrado, de sus necesidades, y todo lo que se quiera compartir al respecto.

\section{Actividades de expresión corporal y de contacto}

La expresión corporal nos ayudará también a relajar el cuerpo, a desentumecerlo, a darle movilidad y apertura, a fortalecerlo, a sentirse vivas y activas. A darse cuenta de que tienen libertad de acción y movimiento tanto corporal como físico en el espacio, a retomar conciencia sobre su propio cuerpo y las decisiones que van unidas a él. Trabajaremos individualmente y por parejas, para ir creando vínculos de confianza con las otras mujeres y de compromiso en el proceso. Es muy importante crear momentos de contacto físico tanto individual como con otra persona, dando lugar a nuevas experiencias que puedan asociarse al cariño, al cuidado, al mimo, a la ternura, al respeto. Tenemos que ser conscientes de las dificultades que pueden tener estas mujeres en el contacto físico, con lo que tendremos que ser muy cuidadosas y prudentes a la hora de realizar los ejercicios e ir siempre a un ritmo donde ellas se encuentren cómodas y seguras.

Todo ello se realizará centrándose en la música, en vivo o grabada, propuesta por la musicoterapeuta. Una escucha activa que nos sugiera y ayude al movimiento, a la expresión corporal y a la reconquista de su cuerpo. 


\section{Audición de canciones y análisis lírico}

Este es un recurso muy utilizado por cantidad de autoras que han hecho terapia con mujeres (Cassity y Theobold, 1990; Whipple y Lindsey, 1999; Curtis, 2012). Es una actividad muy completa donde poder trabajar varios objetivos. La elección musical se hará entre todas, partiendo de una base que la musicoterapeuta planteará y que irá completándose según las peticiones y sugerencias de las participantes, a las que se incluirá y animará a participar en este proceso de selección y decisión. Haremos el trabajo desde una perspectiva feminista de análisis de las letras, de su significado y de lo que nos transmiten, tratando temas relacionados con la violencia hacia la mujer, la figura de la mujer en el contexto sociopolítico, o qué es ser mujer en esta sociedad, entre otras temáticas. Cantaremos las canciones propuestas tanto con la grabación como con la música en directo interpretada por la musicoterapeuta. Se intentará que todas las canciones sean compuestas y/o cantadas por mujeres, ayudando a romper el silencio y el tabú que supone hablar del maltrato y la violencia que han sufrido. Escuchar los testimonios y las experiencias de otras mujeres les ayudará a sentir que no están solas, y facilitará la apertura y expresión de sus sentimientos y pensamientos. Este puede ser un primer paso para la composición de canciones que explicaremos más adelante.

Dentro de la audición de canciones también utilizaremos la modalidad del dibujo para expresar lo que nos sugiere la música, cómo nos hemos sentido y las emociones que nos ha evocado durante su escucha.

\section{Improvisación musical}

La improvisación es un ejercicio verdaderamente importante de comunicación y expresión, que nos puede ayudar mucho a que las mujeres liberen y reconozcan sentimientos y emociones. Es un trabajo libre donde buscamos fluir, exteriorizar, comunicar cómo están, liberar su creatividad, y todo desde la música. Es una forma más pura e inconsciente que nos ayuda a disminuir bloqueos o barreras que de otra forma, normalmente verbal, se hacen muy presentes, sobre todo teniendo en cuenta que estas mujeres hablan de abusos y maltratos, de unos recuerdos traumáticos que son muy difíciles de exteriorizar. Es una actividad que se puede hacer grupal o individual, desde la improvisación con instrumentos o la improvisación con la voz. Da la oportunidad a cada participante de expresarse, de sentirse escuchada, de sentirse valorada, de reconstruir su autoestima y de empoderarse, además de la posibilidad de interaccionar entre ellas con diálogos musicales, y crear cohesión de grupo. En mujeres con tantos problemas de autoestima y confianza en sí mismas, puede ser una actividad intimidante al principio. La musicoterapeuta puede crear una base rítmica o melódica donde ellas vayan introduciéndose, asentando así la base de la improvisación musical, y a partir de ahí combinar las intervenciones de las participantes, que incluso puede ser por grupos o parejas hasta llegar a crear un ambiente de suficiente confianza para hacerlas individuales y expresar sus emociones. Según se vayan desarrollando las sesiones, podemos pedir que sea alguna de ellas quien asiente la base rítmica y las demás vayan uniéndose, trabajando la iniciativa, la asertividad, y mostrando nuestro apoyo y escucha hacia esa participante. Empezaremos con improvisación instrumental ya que la vocal puede generar más resistencia al principio, especialmente en mujeres que han sido siempre silenciadas, pero iremos incluyéndolo poco a poco ya que es un trabajo realmente eficaz para que descubran y alcen su voz.

Se pueden hacer improvisaciones rítmicas y/o melódicas, bien partiendo de una canción que se esté trabajando o como algo nuevo y espontáneo que surja durante la sesión. Es importante que haya una buena selección de instrumentos para que cada mujer pueda encontrar alguno que resuene con ella y con el que se encuentre cómoda. 


\section{Composición de canciones (Songwriting)}

El proceso de escribir una canción personal es una actividad excelente para ponerle nombre y forma a todos esos sentimientos y sensaciones que tienen y que necesitan expresar. Al igual que el análisis de letras en canciones, ha sido utilizado por muchos musicoterapeutas que han trabajado en este campo (Cassity y Theobold, 1990; Whipple y Lindsey, 1999; Curtis, 2012; Fernández, 2010). Una manera de autoconocimiento, de exteriorizar, contar y liberarse de sus experiencias de violencia, pero también una manera de mirar al futuro y poner en palabras lo que quieren para la nueva vida que empiezan. El trabajo profundo y personal que se hace al componer una canción propia y lo que ello conlleva - exploración personal, expresar y contar sus vivencias, cantar y hacerse oír, ser escuchada y comprendida, activar su creatividad, tomar decisiones- puede evocar emociones y sentimientos muy profundos, poderosos y de gran ayuda para el aumento de la autoestima y la satisfacción personal.

La composición puede ser a partir de una canción que les guste y que cambiemos su letra con la escrita por la propia participante, o podemos componer la música y la letra a partir, por ejemplo, de patrones que hagan en sus improvisaciones y de interacciones musicales que surjan en la sesión. La composición puede ser de nuevo, colectiva, personal o incluso para alguien, dependiendo de cómo se esté desarrollando el grupo y de lo que tengamos como objetivo. Al final del proceso creativo, es importante grabar las canciones que se hayan hecho, no solo para que quede reflejado el trabajo que han realizado y que se puedan llevar su canción en un $\mathrm{CD}$ o cualquier medio tecnológico, sino también y lo más importante, para que se oigan y escuchen su voz, para que cuenten y no se avergüencen de lo que han pasado, para que nunca olviden quienes son y que quieren, para que no se dejen silenciar de nuevo, para empoderarse y recuperar su identidad propia.

\section{Recrear, Cantar y tocar}

Tanto cantar como tocar instrumentos será la base de nuestras sesiones de musicoterapia, ser capaces de expresar y hacerse oír a través de cualquier interacción musical. Sabemos que el canto y la participación instrumental nos dan las herramientas para que se descubran ellas mismas, para que encuentren y manifiesten su voz, se expresen y comuniquen, se escuchen y valoren su discurso, canalicen sus emociones y energías, para que sean conscientes de quienes son y de lo mucho que tienen que aportar al mundo. La unión de las voces y los instrumentos en grupo, hará que se sientan acompañadas en sus experiencias, que rompan el silencio y el aislamiento al que han estado sometidas, que sean capaces de expresarse y sentirse escuchadas, que se cree cohesión en el grupo y sororidad.

A nivel instrumental añadiremos el llamado círculo de tambores (drum circle), un potente canalizador de energías y de contacto con nuestro yo interior más básico. El sonido profundo y la demanda física que conllevan estos instrumentos ayudará a soltar el enfado, la ira y la rabia, y por otro lado a coger seguridad en sí mismas, a fortalecerse (física, psicológica y espiritualmente) y a empoderarse.

\section{CONCLUSIONES}

Como hemos venido hablando durante todo el estudio, la música es un poderoso vehículo para el autoconocimiento, para la expresión y para canalización de emociones. Ofrece la posibilidad a estas mujeres a encontrase, escucharse $\mathrm{y}$ hacerse escuchar, sentirse comprendidas y acompañadas, fortalecerse y recuperar su poder personal, su autoestima, su 'Yo' interno, crear su nueva imagen de identidad. Les ayudará a reconocer y nombrar todas 
esas sensaciones y sentimientos, plasmarlos en cada momento musical, y así poder superar poco a poco las experiencias traumáticas y abusivas que han vivido.

A través de la música se facilita el derribe de las barreras comunicativas que puedan tener verbalmente, las barreras para relacionarse con otras personas, rompiendo así el aislamiento social al que han sido sometidas durante mucho tiempo. Lo que queremos conseguir es que estas mujeres tengan una buena calidad de vida, puedan restaurar sus capacidades básicas de identidad, confianza, autonomía, iniciativa y desarrollar una vida propia.

A esto añadir el trabajo musicoterapéutico que se haría con los menores, de introspección, asimilación y reconocimiento de sentimientos, y expresión a través de la música. La música, no sólo como canal comunicativo, sino como canal educativo y de reorganización de conductas y aprendizajes.

Todo ello daría lugar a un bonito reencuentro individual-personal y por supuesto a un bonito reencuentro entre madres y menores para recuperar y fortalecer sus lazos afectivosemocionales.

Cada vez empiezan a realizarse más trabajos de musicoterapia con mujeres que han sufrido violencia de género en la pareja, pero son muy pocos los trabajos realizados con los menores que se ven expuestos a la violencia de género familiar, por lo que es un campo que necesita estudio e investigación.

\section{REFERENCIAS}

Achenbach, T.M. (1993). Empirically based taxonomy: How to use syndromes and profile types derived from the CBCL/4-18, TRF and YSR. University of Vermont: Burlington.

Amor, P.J., Echeburúa, E., de Corral, P., Zubizarreta, I. y Sarasua, B. (2002). Repercusiones psicopatológicas de la violencia doméstica en la mujer en función de las circunstancias del maltrato. International Journal of Clinical and Health Psychology, 2(2),227-246. Recuperado de: https://www.redalyc.org/articulo.oa?id=337/33720202

Annesley, L. (2018). Music therapy within an integrated project for families exposed to domestic violence: A qualitative study of professionals 'perspectives. British Journal of Music Therapy, 32(2), 96-104. DOI: 10.1177/1359457518805281

Barreiro, A.M (2018). Intervención de Enfermería en Atención Plena para Víctimas de Violencia de Género en el Ámbito Íntimo. IV Congreso Virtual Internacional de Enfermería en Salud Mental.

Belloch, A., Sandín, B. y Ramos, F. (2009). Manual de psicopatología, II. España: Mc Graw Hill.

Boletín Oficial del Estado (BOE) núm, 313, de 29 de diciembre de 2004. Ley Orgánica 1/2004, de 28 de diciembre, de Medidas de Protección Integral contra la Violencia de Género

Boletín Oficial del Estado (BOE) núm. 175, de 23 de julio de 2015. Ley Orgánica 8/2015, de 22 de julio, de modificación del sistema de protección a la infancia y a la adolescencia. 
Bruscia, K. (1997). Definiendo musicoterapia. Salamanca: Amarú.

Campbell J., Snow, A., Dienemann J., Kub, J., Schollenberger, J., O’Campo, P., Carlson, A. y Wynne, C. (2002) Intimate Partner Violence and Physical Health Consequences. Arch Intern Med. 162(10):1157-1163. doi:10.1001/archinte.162.10.1157

Cassity, M. D., \& Theobold, K. A. (1990). Domestic violence: Assessments and treatments employed by music therapists. Journal of Music Therapy, 27(4), 179-194.

Curtis, S.L. (2015). Music Therapy for Women Who Have Experienced Domestic Violence. The Oxford Handbook of Music Therapy. DOI: 10.1093/oxfordhb/9780199639755.013.30

Curtis, S. L. (1996). Singing subversion, singing soul: Women's voices in feminist music therapy. (tesis doctoral). Concordia University, Montreal, Canadá.

Curtis, S.L. (2012). Women survivors of abuse and developmental trauma. En Ken Bruscia (Ed.), Guidelines for Music Therapy Practice. 8 (pp.1-33) Philadelphia, PA: Barcelona Publishers.

Delegación del Gobierno contra la Violencia de Género. Ministerio de la Presidencia, Relaciones con las Cortes e Igualdad. En https://violenciagenero.igualdad.gob.es/violenciaEnCifras/home.htm

DSM-V (2014). Manual diagnóstico y estadístico de los trastornos mentales. Asociación Americana de Psiquiatría (APA). España: Editorial Médica Panamericana.

Echeburúa, Enrique y de Corral, P. (1998). Manual de violencia familiar. Madrid: Siglo XXI.

Expósito, F. (2011). Violencia de Género. Mente y cerebro, (48), 20-25. Recuperado de https://www.investigacionyciencia.es/revistas/mente-y-cerebro/redes-sociales-527/violenciade-gnero-8894

Expósito, F. (2012). Efectos psicosociales de la violencia de género sobre las víctimas directas e indirectas: prevención e intervención. Ministerior de Sanidad, Servicios sociales e Igualdad. $\quad$ Recuperado de https://www.inmujer.gob.es/areasTematicas/estudios/estudioslinea2012/docs/Efectos_psic osociales_violencia_Web_853.pdf

Felitti, V.J., Anda, R.F., Nordenberg, D., Williamson, D.F., Spitz, A.M., Edwards, V., Koss, M.P. and Marks, J.S. (1998). Relationship of Childhood Abuse and Household Dysfunction to many of the Leading Causes of Death in Adults. American Journal of Preventive Medicine. 14(4), 245-258. DOI: 10.1016/S0749-3797(98)00017-8

Fernández, D. (2015a). Destrucción y reconstrucción de la identidad de mujeres maltratadas:análisis de discursos autobiográficos y de publicidad institucional (tesis doctoral). Ministerio de Sanidad, Política Social e Igualdad. Centro de Publicaciones.

Fernandez, T. (2004). Violencia contra la mujer en México. Comisión Nacional de los Derechos Humanos. México D.F. 
Fernández, T. (2013) La musicoterapia al rescate de la identidad de género en mujeres violentadas por su cónyuge. El colegio de la frontera norte (Ed.), Fronteras culturales, alteridad y violencia (pp.317-353). México.

Fernández, T. (2006). "Hacia un nuevo camino":programa de investigación-acción sobre autoestima y musicoterapia con mujeres violentadas. Enseñanza e Investigación en Psicología, 11(1), 65-79.

Fernández, T. (2010). Musicoterapia y género: Una nueva visión en el trabajo de reconstrucción de la identidad en mujeres que padecen violencia doméstica y ante determinadas situaciones de salud. Sistema Mexicano de Investigación en Psicología. Recuperado de: https://www.academia.edu/1967588 Musicoterapia_y_género_Una_nueva_visión_en_el_trabajo_de_reconstrucción_de_la_ide ntidad_en_mujeres_que_padecen_violencia_doméstica_y_ante_determinadas_situaciones _de_salud_2010_

Fernández, T. (Abril de 2015a). Psicología Transcultural. Haciendo un trueno de mi herida: mujeres violentadas, identidad cultural y de género y musicoterapia. pp. 89-94. XLII Congreso Nacional de Psicología. Tijuana, México.

Fernández, T. (2015b). Estudio de la ansiedad antes y después de la aplicación de musicoterapia en mujeres violentadas de cuba y de méxico. Comparación y perspectivas culturales. Integración Académica en Psicología, 3(8), 64-75. ART 7

Fernández, M.C.,Herrero, S., Buitrago, F., Ciurana, R., Chocaron, L., García, J., Montón, C., Redondo, M.J. y Tizón, J.L. (2003). Violencia doméstica. Madrid: Ministerior de Sanidad y Consumo.

Galego, V. (2015). Autonomía personal y afrontamiento en mujeres en situación de maltrato (premio tesis doctoral). Ministerio de Sanidad, Servicios Sociales e Igualdad.

Galán, A. (2015). La teoría del apego: confusiones, delimitaciones conceptuales y desafíos. Revista Asociación España de Neuropsiquiatría.36(129), 45-61. DOI: 10.4321/S021157352016000100004

Gómez, I. (2018). Hijas e hijos víctimas de la violencia de género. Aranzadi Doctrinal, 8. Recuperado de http://www.congreso.es/docu/docum/ddocum/dosieres/sleg/legislatura_12/spl_25/pdfs/17. pdf

Hernández-Ruiz, E. (2005). Effect of Music Therapy on the Anxiety Levels and Sleep Patterns of Abused Women in Shelters. Journal of Music Therapy. XLII (2), 140-158. DOI: 10.1093/jmt/42.2.140

Hirigoyen, M. F. (2006). Mujeres maltratadas. Los mecanismos de la violencia en la pareja. Barcelona: Paidós.

Holden, G.W. (2003). Children Exposed to Domestic Violence and Child Abuse: Terminology and Taxonomy. Clinical Child and Family Psychology Review, 6(3), 
Jauset, J.A (2013) Cerebro y música, una pareja saludable. Las claves de la neurociencia musical. Almería:Círculo Rojo.

Kleckner, C. (productora), Khan, A.y Lawrence, J.(directores).(2017) A better man. [Documental]. Canadá: Intervention Productions.

Labrador, F. J., Rincón, P. P., De Luis, P. y Fernández-Velasco, R. (2004). Mujeres víctimas de la violencia doméstica. Programa de actuación. Madrid: Psicología Pirámide.

Lago, P. (2012). La musicoterapia en el trabajo personal de mejora de un grupo de mujeres maltratadas. En Musicoterapia y Salud (Ed.), IV Congreso Nacional de Musicoterapia (pp.59-79). Madrid.

Lago, P. (Septiembre de 2020). Los perversos no tienen canciones: la musicoterapia como modelo terapéutico para mujeres maltratadas, III Jornadas de psicología y música por la UNED, Madrid.

Lorente, M. (2001). Mi marido me pega lo normal. Barcelona: Crítica

López, J. (2016). El síndrome de la mujer maltratada y su relación con las emociones y los procesos educativos de sus hijos (tesis doctoral). Universidad de León, España.

López, R. ( 28 de Junio, 2015). La música como terapia de apoyo para mujeres maltratadas. [Transmisión de radio] Género e Igualdad. Canal UNED, Madrid.

https://canal.uned.es/video/5a6f4a74b1111fe7218b45f9

Ministerio de Sanidad, Servicios Sociales e Igualdad. Portal Estadístico de la Delegación del Gobierno para la Violencia de Género. Recuperado el 5 de Junio de 2020 de: http://estadisticasviolenciagenero.igualdad.mpr.gob.es

Naciones Unidas (1993). Declaración sobre la Eliminación de la Violencia contra la Mujer. Oficina del Alto Comisionado para los Derechos Humanos (ACNUDH). En https://www.ohchr.org/sp/professionalinterest/pages/violenceagainstwomen.aspx

Nieto, L.(Redactora- Locutora).(17 de Septiembre, 2012). Musicoterapia y violencia de género. $1^{*}$ parte. [Transmisión de radio] Canal UNED. Madrid: Formación continua en Radio 3. Recuperado de https://canal.uned.es/video/5a6f2f4ab1111f4e7f8b49d4

Nieto, L. (Redactora-Locutora). (4 noviembre, 2019). A vueltas con la violencia de género. [Transmisión de radio] Canal UNED, Madrid: Formación continua en Radio 3. Recuperado de https://canal.uned.es/video/5db985505578f2342b1b97bc

Nieto, L. (Redactora-Locutora).(18 de Noviembre,2019). Violencia de género: revictimizacion institucional. [Transmisión de radio] Canal UNED, Madrid: Formación continua en Radio 3. https://canal.uned.es/video/5dcbeca45578f2669169cde2

Nieto, L. (Redactora-Locutora). (13 de Enero, 2020). Los hijos como víctimas de violencia de género. [Transmisión de radio] Canal UNED, Madrid: Formación continua Radio 3. 
https://canal.uned.es/video/5e1591775578f210073d5c88

OMS. Recuperado el 27 de junio de 2020 de:

https://www.who.int/es/news-room/fact-sheets/detail/violence-against-women

https://www.who.int/topics/gender based violence/es/

Pasiali, V. (2013). A Clinical Case Study of Family-Based Music Therapy. Journal of Creativity in Mental Health, 8:249-264. DOI: 10.1080/15401383.2013.821925

Perry, B.D. (2001). The neurodevelopmental impact of violence in childhood. En D. Schetky and E.P.Benedek (Ed.). Child and Adolescent Forensic Psychiatry, 18 (pp. 221-238). Washington, D.C: American Psychiatric Press.

Pinheiro, P.S. (2010). Informe Mundial sobre la violencia contra los niños y niñas. ONU. Recuperado de https://www.observatoriodelainfancia.es/oia/esp/documentos_ficha.aspx?id=2954

Patró, R. y Limiñana, R.M. (2005). Víctimas de violencia familiar: Consecuencias psicológicas en hijos de mujeres maltratadas. Anales de psicología. 21(1), 11-17. Recuperado de https://www.um.es/analesps/v21/v21_1/02-21_1.pdf

Rey, M. (2016). Proyecto de intervención social con mujeres víctimas de violencia de género y sus hijas e hijos (tesis de máster). Universidad de Oviedo, España.

Romero, I. (2015). Guía de intervención en casos de violencia de género. Una mirada para ver. Madrid: Editorial Síntesis

Ruiz-Pérez, I., Blanco-Prieto, P., y Vives-Cases, C. (2004). Violencia contra la mujer en la pareja: determinantes y respuestas sociosanitarias. Gaceta Sanitaria, 18(Supl. 2), 4-12. Recuperado de http://scielo.isciii.es/scielo.php?script=sci_arttext\&pid=S0213$91112004000500003 \& \operatorname{lng}=\mathrm{es} \&$ tlng=es.

Samaniego, J. (2020). Del amor romántico a la mismidad emancipadora. Periféricas. Recuperado de https://perifericas.es/blogs/blog/amor-romantico-feminismo-simone-debeauvoir-alexandra-kollontai

Save the Children (2011). En la violencia de género no hay una sola víctima. Atención a los hijos e hijas de mujeres víctimas de violencia de género. Recuperado de https://www.savethechildren.es/publicaciones/en-la-violencia-de-genero-no-hay-una-solavictima

Suriá, R., Rosser, A.M., Villegas, E. (2014). Efectos de la exposición a la violencia de género en los menores. Psicología jurídica y forense. Investigación-acción, 29-49. Recuperado de https://www.academia.edu/9185524

suriá_rosser_y_villegas_2014_efectos_de_la_exposición_a_la_violencia_de_género_en_los_ menores

Tribunal Supremo. STS núm. 247/2018 de 24 de Mayo. Recuperado de: https://supremo.vlex.es/vid/727894245 
Unicef (2006). Convención sobre los Derechos del Niño. Recuperado de https://www.unicef.es/publicacion/convencion-sobre-los-derechos-del-nino

Walker, L. E. (1979). The battered woman. New York: Harper and Row

Walker, L.E. (2012). El Síndrome de la Mujer Maltratada. Desclée de Brouwer Editores.

Walker, L. E. (2017). Validation of the Battered Woman Syndrome Questionnaire. Simposio llevado a cabo en APA Annual Convention, Washigton D.C. Recuperado de https://www.drlenoreewalker.com/2017-apa-annual-convention/

Whipple, J \& Lindsey, R.S (1999). Music for the Soul: A Music Therapy Program for Battered Women. Music Therapy Perspectives, 17, 61-68.

WFMT. Recuperado el 2 agosto de 2020 de: https://wfmt.info/2011/05/01/announcing-wfmtsnew-definition-of-music-therapy/

Zubizarreta, I. (2004). Consecuencias psicológicas del maltrato doméstico en las mujeres y en sus hijos e hijas. EMAKUNDE, Instituto Vasco de la Mujer. 


\section{ANEXOS}

\section{LISTADO DE CANCIONES}

Esta es una lista de canciones con diferentes temáticas y estilos que se sugieren para el trabajo de análisis lírico y songwriting.

- Ya no más - Susana Ochoa

- Gracias a la vida - Violeta Parra

- La vida es un Carnaval - Isaac Delgado interpretada por Celia Cruz

- Ojalá - Silvio Rodriguez

- Que no que no - Rozalén

- La puerta violeta - Rozalén

- Contigo - La otra

- Mujer Lunar - Rebeca Lane

- Quisiera olvidarme de tu nombre - Rebeca Lane

- Quien manda - Mala Rodriguez

- Maria se bebe las calles - Pasión Vega

- Ella - Bebe

- Que nadie - Malú

- I will survive - Gloria Gaynor

- Aguante - Mala Rodriguez

- La batalla invisible - Mabel Flores

- Pa' respirar - Bomba Estereo 